\section{Directional sliding of water: biomimetic snake scale surfaces}

\author{
Yizhe Zhao ${ }^{1}$, Yilin $\mathrm{Su}^{1,2}$, Xuyan $\mathrm{Hou}^{2}$ and Minghui Hong ${ }^{1 *}$
}

\begin{abstract}
Bioinspired superhydrophobic surfaces have attracted many industrial and academic interests in recent years. Inspired by unique superhydrophobicity and anisotropic friction properties of snake scale surfaces, this study explores the feasibility to produce a bionic superhydrophobic stainless steel surface via laser precision engineering, which allows the realization of directional superhydrophobicity and dynamic control of its water transportation. Dynamic mechanism of water sliding on hierarchical snake scale structures is studied, which is the key to reproduce artificially bioinspired multifunctional materials with great potentials to be used for water harvesting, droplet manipulation, pipeline transportation, and vehicle acceleration.
\end{abstract}

Keywords: biomimetic; hierarchical micro/nanostructures; directional superhydrophobicity; anisotropic friction

Zhao YZ, Su YL, Hou XY, Hong MH. Directional sliding of water: biomimetic snake scale surfaces. Opto-Electron Adv 4, 210008 (2021).

\section{Introduction}

Many species in nature, such as plants ${ }^{1-4}$, insects ${ }^{2,4-9}$, reptiles $^{10-11}$, and marine organisms $s^{4,12}$, exhibit remarkable surface functions. These precious properties are evolved over millions of years through natural selection, enabling species to thrive in extreme environments. It is well known that the vast majority of extraordinary surface properties derive from their unique hierarchical morphological features, ranging from a few tens of nanometers to hundreds of micrometers in size.

Inspiration from natural functional structures, especially famous water repellent plant leaves, like lotus leaves, a variety of artificial superhydrophobic surfaces with liquid-repellent properties have been designed and fabricated through the uniquely constructed geometrical micro/nanostructures and the chemical composition of the surface ${ }^{1-6}$. The artificial liquid-repellent surfaces ex- hibit certain remarkable wetting characteristics originating from a high water contact angle (CA) greater than $150^{\circ}$ and a small water sliding angle (SA) less than $10^{\circ}$ are of great value for a variety of applications, such as water collection ${ }^{6}$, self-cleaning ${ }^{13}$, anticorrosion ${ }^{14}$, oil/water separation ${ }^{15}$, antifreezing ${ }^{16}$, anti-fogging ${ }^{17}$, and friction reduction ${ }^{18}$. Several techniques have been developed to construct superhydrophobic surfaces, such as chemical vapor deposition ${ }^{19}$, electrospinning ${ }^{20}$, lithography ${ }^{21}$, and laser fabrication ${ }^{22-25}$. Up to now, despite the fact that artificial superhydrophobic surfaces have already revealed a significant role in both fundamental research and practical applications, continued efforts in endowing novel materials with unique properties are still highly required to realize their structural and functional integrity.

Compared with traditional surfaces with hydrophobic property, the manufacture of superhydrophobic surfaces

${ }^{1}$ Department of Electrical and Computer Engineering, National University of Singapore, 4 Engineering Drive 3, 117576, Singapore; ${ }^{2}$ State Key Laboratory of Robotics and System, Harbin Institute of Technology, Harbin 150001, China..

*Correspondence: MH Hong, E-mail: elehmh@nus.edu.sg

Received: 21 January 2021; Accepted: 28 February 2021; Published online: 6 April 2021

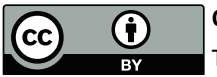

Open Access This article is licensed under a Creative Commons Attribution 4.0 International License.

To view a copy of this license, visit http://creativecommons.org/licenses/by/4.0/.

(C) The Author(s) 2021. Published by Institute of Optics and Electronics, Chinese Academy of Sciences. 
with unique versatility has broad applications and has become a hot topic in the field of surface technology. Constructing functional superhydrophobic surfaces with high stability, versatility, flexibility, and universality have a high practical value ${ }^{26}$. For instance, Cao et al. designed a 2D MXene-reinforced robust liquid-repellent surface with self-cleaning and photothermal-actuating binary effects ${ }^{27}$. Song et al. reported a droplet-based self-propelled autonomous device composed of superhydrophobic miniboat with superhydrophilic pore ${ }^{28}$. Wang et al. acquired a wearable superhydrophobic elastomer skin with switchable wettability to switch wetting states, which enables the surface to capture and release multiple droplets in paralle ${ }^{29}$. Furthermore, some researchers have conducted research on liquid transportation. Paradisanos et al. demonstrated a methodology to fabricate a gradient wettability pattern on Si, exhibiting a high velocity for liquid spreading on a surface tension gradient in ambient conditions ${ }^{30}$. Kirner et al. reproduced the shape of the micrometre-sized tilted spikes found underneath the wings of the bark bug D. magnus, on steel surfaces to realize fast unidirectional fluid transport ${ }^{31}$. Wu et al. proposed a strategy for horizontal vibration for multimode, large-volume-range, and high-speed unidirectional microdroplet manipulation, which is ascribed to the rolling state on superhydrophobic slant microwall arrays fabricated by the femtosecond laser ${ }^{32}$. These pioneering works demonstrated the feasibility of superhydrophobic surfaces with unique multifunctionality. Therefore, developing practical and effective methods for manufacturing and functionalizing superhydrophobic surfaces will be beneficial in improving the practicability of superhydrophobic surfaces in various technical applications.

In this work, the directional transportation directivity of the bionic snake scale-like superhydrophobic surface has been reported for the first time. It was inspired from the unique superhydrophobicity and frictional anisotropy properties of the snake scales, which are promising for a wide range of scientific fields ${ }^{10-11}$. These properties are primarily due to the unique hierarchical morphological feature of the snake scales. Fabrication of snake scale-like hierarchical micro/nanostructured surfaces with unique superhydrophobicity is of great interest from the viewpoint of both experimental research and practical applications. Herein, a bionic snake scalelike superhydrophobic stainless steel surface with these feats via laser processing is reported. The study shows a novel triple inclined-beam laser ablation (IBLA) treatment at oblique incidence is implemented to fabricate the surface with three-dimensional inclined bionic snake scales that can effectively achieve the anisotropic friction. Additionally, the synergistic effect of hierarchical micro/nanostructures and the intrinsic change in the surface chemistry with the exposure to air after the laser irradiation exhibits unique superhydrophobicity along a particular direction. These characteristics collectively enable the fantastic synergy: directional water transportation. The results prescribe a method for reproducing these capabilities of snake scales in artificial materials. The technology is flexible, chemical-free, and can be easily implemented through commercial industrial laser processing systems. Accordingly, directional water transportation materials can be useful in a wide range of applications including water harvesting, droplet manipulation, pipeline transportation, and vehicle acceleration.

\section{Materials and methods}

\section{Material}

The experiment is performed on 316L stainless steel substrates with a thickness of $2 \mathrm{~mm}$. Before laser ablation, the $316 \mathrm{~L}$ stainless steel plates $(50 \mathrm{~mm} \times 50 \mathrm{~mm})$ are polished using $800 \#$ abrasive papers and ultrasonically cleaned in deionized water to remove oxide layers and impurities.

\section{Laser ablation}

The polished samples are ablated by a Q-switched DPSS laser (Model: AVIA LX 355-20-50, Coherent) with a wavelength of $355 \mathrm{~nm}$, pulse duration of $10 \mathrm{~ns}$, and repetition rate up to $50 \mathrm{kHz}$. The output power can be continuously tuned with a variable attenuator. A 3D galvanometric scanner is employed to move the laser beam along $X$-, $Y$ - and, $Z$-directions over a sample surface. The laser is focused normally and obliquely incident $\left(45^{\circ}\right)$ onto the sample surfaces through an f-theta lens, producing a focal spot diameter of about $30 \mu \mathrm{m}$. Programmable patterns including snake scale structures textures, randomly distributed nanoparticles, and periodic inclined cone-shaped structures are generated on the pretreated plate surfaces by laser with different laser powers $(4.2 \mathrm{~W}, 0.5 \mathrm{~W}$, and $0.5 \mathrm{~W})$, different repetition rates $(50 \mathrm{kHz}, 30 \mathrm{kHz}$, and $30 \mathrm{kHz})$, different scanning times (1000 times, 1 time, and 1 time), and different scanning speeds $(50 \mathrm{~mm} / \mathrm{s}, 1 \mathrm{~mm} / \mathrm{s}$, and $1 \mathrm{~mm} / \mathrm{s})$ by different steps 
of the laser processing, respectively. Moreover, the scanning space of the second and third steps are both $30 \mu \mathrm{m}$. Then, the textured substrates are ultrasonically cleaned in deionized water for $10 \mathrm{~min}$ to remove the floating steel powder on the surface and put in the ambient air for a long time without any chemical modification treatment.

\section{Results and discussion}

Stainless steel, which is one of the most commonly used materials in today's society, is chosen as a substrate material for superhydrophobic surface fabrication due to its outstanding properties, such as thermal stability, chemical resistance, nontoxicity, and high mechanical strength. Laser precision engineering, which has already been proved as a valuable, versatile, and reliable technique in the fabrication of biomimetic structures and devices that feature natural materials due to its advantages of rapid, mask-free, chemical-free, and large-area micro-nanofabrication $^{33-38}$. In this work, we use the new IBLA method to fabricate periodically bionic snake scale-like structured stainless steel surfaces. A Q-switched DPSS laser is employed for the processing. By using the IBLA method, the pulsed laser beam is in oblique incidence on the sur- face of stainless steel. Different from the conventional laser processing, the oblique beam ablation is prone to induce some unique hierarchical bionic structures. In order to realize the surface morphology of snake scales on the stainless steel surface for acquiring the unique superhydrophobicity and frictional anisotropy properties of the snake scales, we need to understand the surface morphology of snake scales in details. Figure 1(a) shows a bird's-eye-view image of the West African Gaboon Viper (Bitis rhinoceros), which has different morphology and mechanical properties of the scales from different body sections (leading body half, middle trunk, and tailing body half) and positions (dorsal, lateral, and ventral). According to the existing literature reports, dorsal scale surfaces of Bitis rhinoceros has a high static CA beyond $160^{\circ}$ and low SA below $20^{\circ}$ (Fig. 1(b) $)^{10}$. As shown in Fig. 1 (c), a tilted-view scanning electron microscopy (SEM) image of this dorsal scale surfaces of Bitis rhinoceros shows a hierarchical micro/nanostructure consisting of microscopic inclined cone-shaped structures. Therefore, inspired by this special hierarchical micro/nanostructure, a highly bionic snake scale-like three-level hierarchical micro/nanostructured surface via the IBLA method is
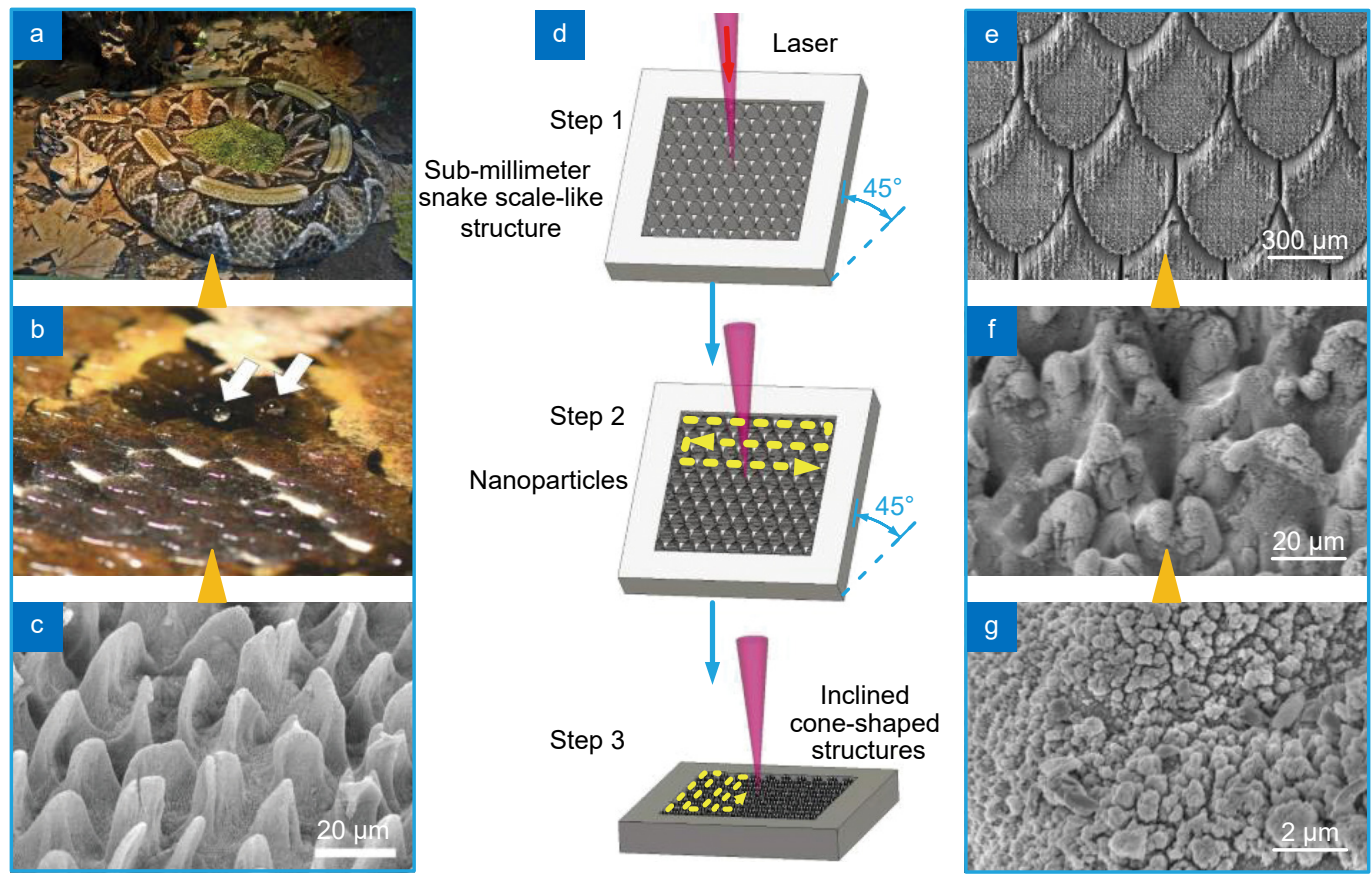

Fig. 1 | Schematic illustration and fabrication process of a snake scale-like surface via the IBLA method. (a) The photographs of the West African Gaboon Viper (Bitis rhinoceros). (b) Photographic image of the dorsal skin of a living individual of Bitis rhinoceros after sprinkling with water. Figure reproduced with permission from ref. ${ }^{10}$, Copyright 2014, PLOS Publishing. (c) SEM image of the hierarchical structures of the dorsal scale surfaces of Bitis rhinoceros. Figure reproduced with permission from ref. ${ }^{10}$, Copyright 2014, PLOS Publishing. (d) Schematic illustration of the manufacture of snake scale-like artificial surface by IBLA technique. (e-g) SEM images of the as-prepared sample surfaces at different magnifications. 
thus fabricated. The principle and preparation process of the snake scale-like artificial surface are shown in Fig. 1(d). First, the sub-millimeter snake scale-like structure array is fabricated on the stainless steel by nanosecond laser oblique ablation. In order to introduce anisotropy into the snake scale-like structure array, the snake scalelike structure array is designed to be tilted cross stacked arrangement as same as the real snake skin, and the distances between adjacent snake scales in the transverse and longitudinal directions are designed to be different. Then, the nanoscale nanoparticles and the microscale inclined cone-shaped structures are ablated on each unit of snake scale-like structure array by the second and third steps of laser processing for acquiring superhydrophobicity and promoting anisotropy. As a result, the highly bionic three-level hierarchical snake scale-like structure array can be achieved. Figures $1(\mathrm{e})-1(\mathrm{~g})$ show the SEM images of the as-prepared sample surface morphology with different magnifications. The high magnification SEM images indicate that the size of the unit of snake scale-like structure array is about $500 \mu \mathrm{m}$ and the surface of the unit is rather rough, composed of inclined cone-shaped microstructures. It is worth noting that the surfaces of these microstructures are not smooth, with plenty of nanoparticles that are densely and unevenly distributed on the surface of each micro-cone. These sub-millimeter, micro- and nanoscale structures are crucial for obtaining directivity and superwetting characteristics that are similar to what is observed in dorsal scale surfaces of Bitis rhinoceros.

To quantificationally investigate the influence of the three-level hierarchical structures of the snake scale-like surfaces on directional superhydrophobicity, liquid-repellent property measurement has been carried out for the laser fabricated snake scale-like surfaces with different hierarchical structures, and the sliding property of the as-prepared surfaces has been tested both along and against the snake scale direction. Each measured value is averaged over three locations. As shown in Fig. 2, the SEM images of micro/nanostructured snake scale-like surfaces with different hierarchical structures at different amplified scales have been shown. It can be seen that the overall surfaces are periodic arrangement of snake scales and oblique grooves with different hierarchical structures by one step, two steps, and three steps of the laser precision engineering, respectively (Figs. 2(a), 2(d) and $2(\mathrm{~g})$ ). Enlarged images demonstrate that the surface roughness of the as-prepared samples becomes larger with the increase of processing steps (Figs. 2(b), 2(e) and $2(\mathrm{~h})$ ), and through the three steps of the laser processing, the microscale inclined cone-shaped structures are created on each unit of the snake scale-like structure array. As shown in Figs. 2(c), 2(f) and 2(i), the surface is gradually adhered by more and more particulate melt protrusions with the increase in processing steps, and the size of particulate melt protrusions getting smaller and smaller to the nanoscale. These results indicate that the asprepared surface by the three steps of the laser processing have similar three-level hierarchical micro/nanostructures with those on the real snake scale. The surfaces with different hierarchical structures exhibit different wetting properties, and the CA ( $5 \mu \mathrm{L}$ water droplet) measured parallel to surfaces with different hierarchical structures are about $73^{\circ}, 133^{\circ}$, and $159^{\circ}$ as shown in the bottom-left corner of Figs. 2(a), 2(d) and 2(g), respectively. Considering the fact that the $\mathrm{CA}$ of the pristine stainless steel with a flat surface is only about $72^{\circ}$, the IBLA fabricated hierarchical micro/nanostructures are considered to be an essential factor to gain the hydrophobicity. Figure 2(j) depicts the time-dependence of CAs for the as-prepared surfaces with different hierarchical structures by different steps of the laser processing when they are exposed to air. Before the laser treatment, all the bare stainless steel surfaces were hydrophilic. Once ablated by laser, the fresh stainless steel surfaces immediately exhibit hydrophilic or even superhydrophilic behavior, and the water droplet can even fully spread out when it touches the laser-induced rough surface. It is unable to acquire a CA value as the droplet is almost completely penetrated into the grooves. This phenomenon is well pronounced within 10 days after the laser ablation. According to the Wenzel theory, the increase of surface roughness leads to an amplification effect of surface wettability ${ }^{39}$.

$$
\cos \theta_{\mathrm{w}}=r \cos \theta_{\mathrm{f}},
$$

where $r>1$ is surface roughness parameter; $\theta_{\mathrm{f}}$ and $\theta_{\mathrm{w}}$ represent $\mathrm{CAs}$ for the flat and rough surfaces, respectively. Equation (1) reveals that with the enhancement of surface roughness, a hydrophilic surface becomes more hydrophilic and a hydrophobic surface becomes more hydrophobic. Therefore, at early stage of CA evolution, the fresh stainless steel surfaces exhibit very small contact angles due to the increment of roughness. Another reason for the observed superhydrophilicity is the change of surface chemical compositions before/after the laser 

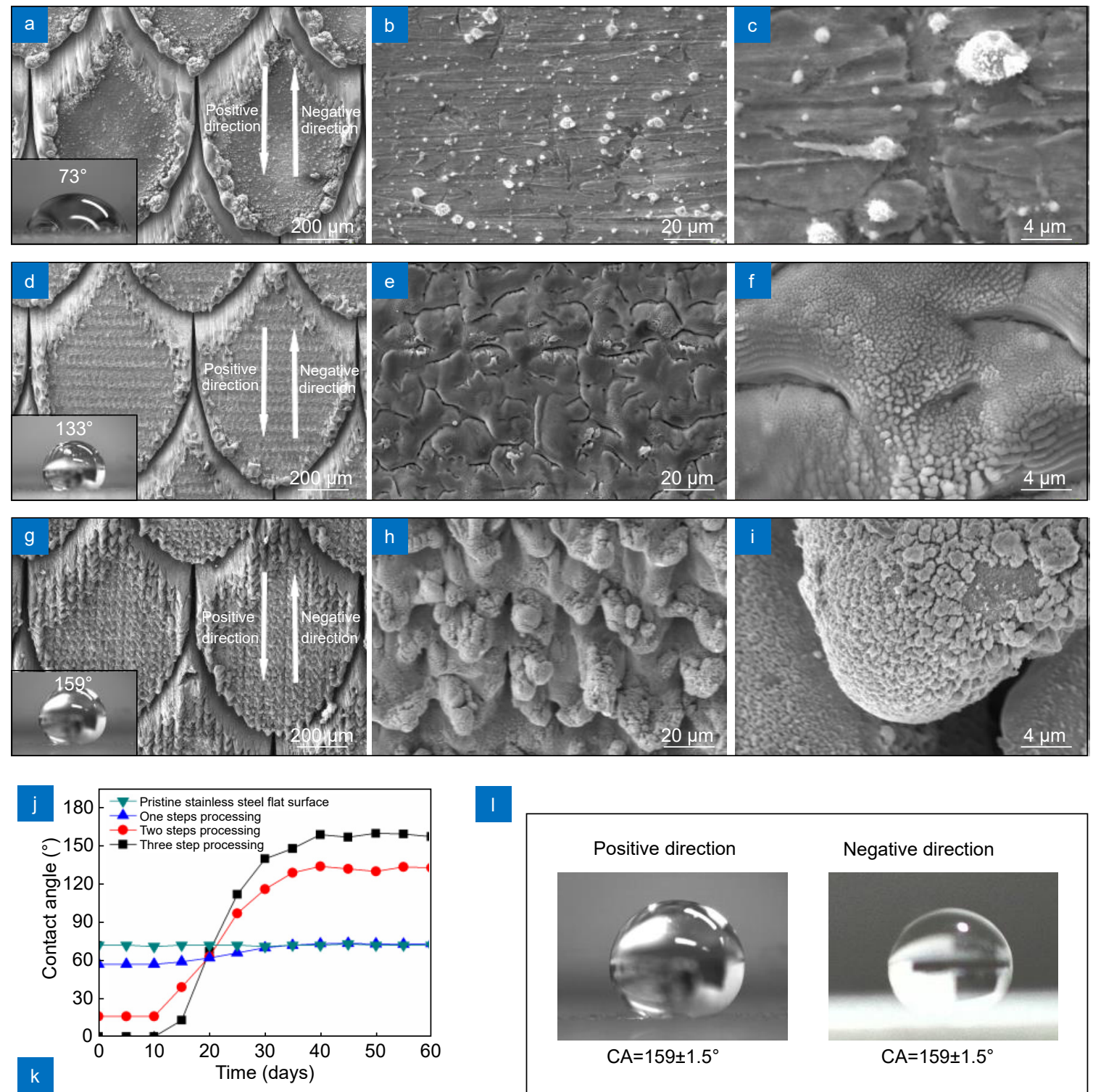

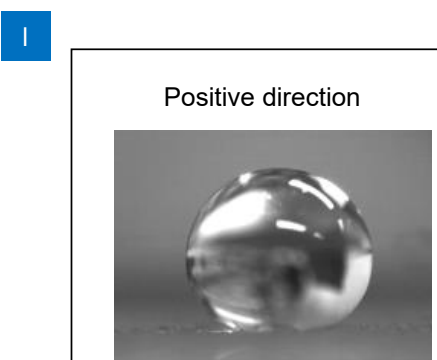

$C A=159 \pm 1.5^{\circ}$

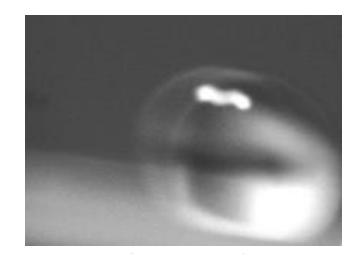

$\mathrm{SA}=7 \pm 1.5^{\circ}$

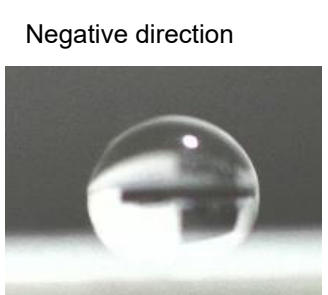

$C A=159 \pm 1.5^{\circ}$

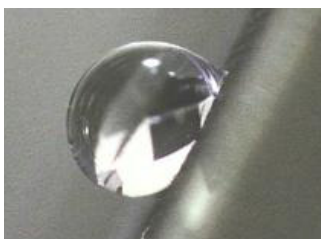

$\mathrm{SA}=81 \pm 1.5^{\circ}$

Directional superhydrophobicity

30

Time (h)

Fig. 2 | Micro/nanostructures, and wetting behavior of the superhydrophobic surfaces with different hierarchical structures by different steps of the laser processing. (a) SEM images of the hierarchical structures of the snake scale-like array by one step laser processing; the inset shows that the CA of the water droplet is $73^{\circ}$. (b, c) SEM images of (a) at different magnified scales. (d) SEM images of the hierarchical structures of the snake scale-like array by two steps laser processing; the inset shows that the CA of the water droplet is $133^{\circ}$. (e, f) SEM images corresponding to (d) at different magnified scales. (g) SEM images of the hierarchical structures of the snake scale-like array by three steps laser processing; the inset shows that the CA of the water droplet is $159^{\circ}$. (h, i) SEM images corresponding to ( $\mathrm{g}$ ) at different magnified scales. (j) Time-dependence of CAs for the hierarchical surfaces by different steps laser processing exposed to air. (k) Superhydrophilicity of the complete three-level hierarchical surfaces in $30 \mathrm{~h}$. (I) Wetting results (CAs and SAs) on the surface corresponding to opposite directions, respectively. 
ablation, which will be explained in detail in the mechanism section later. Observing contact angle curve in Fig. 2(j), it is noted that when the laser ablated surfaces are exposed to air, their surface hydrophilicity decreases and the CA increases over time. Between Day 11 and Day 30, the CA of surfaces by the two steps and three steps of laser processing witnessed a sharp increase from $0^{\circ}$ to $127^{\circ}$ and $0^{\circ}$ to $139^{\circ}$, respectively. It is noted that fresh surfaces reach hydrophobic behaviors. With the time exposed to air increases, only the surface CA by the three steps of laser processing exceeds $150^{\circ}$, and the CA of $159^{\circ}$ is obtained when the three steps laser-induced surface is exposed to ambient air for 40 days. Then, CA gradually remains in a relatively stable state of $159 \pm 2^{\circ}$, which can be characterized as a typical superhydrophobic surface. Thus, it can be concluded that after the laser ablation and exposure to air, only the three steps laser-induced three-level hierarchical micro/nanostructured surface shows superhydrophobicity. Additionally, as shown in Fig. 2(k), the as-prepared surface by the three steps of laser processing retains superhydrophobicity for a long time (at least 30 hours) in contact with water until the droplets on the surface evaporate. Meanwhile, the superhydrophobicity is also maintained by remeasurement at the location where the droplets evaporate.

Like the snake skin, such a snake scale-like three-level hierarchical micro/nanostructured surface displays supe-

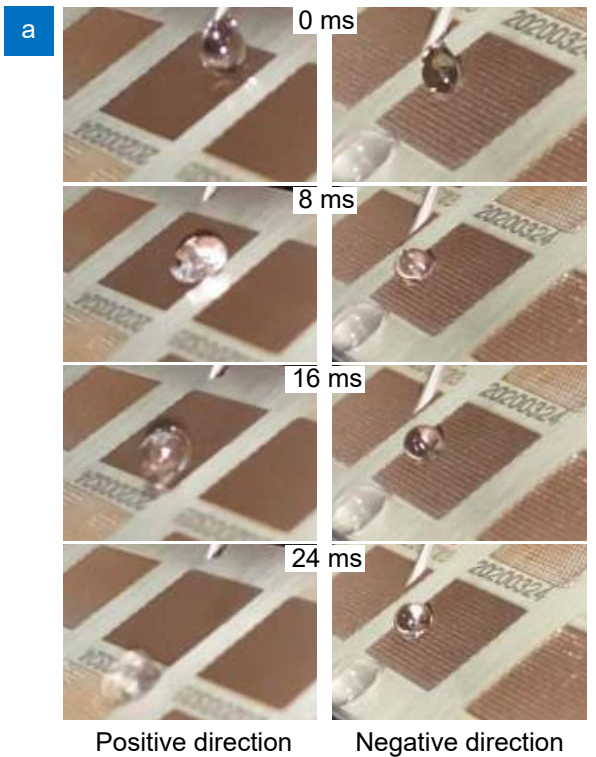

rhydrophobic anisotropic sliding. As shown in Fig. 2(1) and Movie S1 (see Supplementary information), in the negative direction $\left(\mathrm{CA}=159^{\circ} \pm 1.5^{\circ}, \mathrm{SA}=81^{\circ} \pm 1.5^{\circ}\right.$, measured parallel to the surfaces), $\mathrm{SA}$ is larger than that in the positive direction $\left(\mathrm{CA}=159^{\circ} \pm 1.5^{\circ}, \mathrm{SA}=7^{\circ} \pm\right.$ $1.5^{\circ}$, measured parallel to the surfaces), positive direction and negative direction are the measured directions as labeled in Figs. 2(a), 2(d) and 2(g). These results demonstrate that anisotropic superhydrophobic and directional sliding states can be realized by this snake scalelike three-level hierarchical micro/nanostructured surface. Herein, the surface chemical modification has not been utilized in the preparation, which means that the unique three-level hierarchical micro/nanostructures mainly induce the directional wetting performance.

To evaluate the directional control of the water-sliding ability, the sliding process is monitored in real time, the positive and negative directions sliding states of the snake scale-like surface are measured (Fig. 3 and Movies S2 to S5, Supplementary information). As shown in Fig. $3(\mathrm{a})$, the sample is first tilted $30^{\circ}$ along the positive direction, droplets (about $20 \mu \mathrm{L}$ ) are randomly added at different locations, all the droplets instantaneously rolling off the as-prepared surface along the positive direction. Then, the sample turns $180^{\circ}$ to the negative direction and it still tilted at $30^{\circ}$. All the added droplets are adhered on it. The motion of water flowing along the positive and negative directions is also studied when the

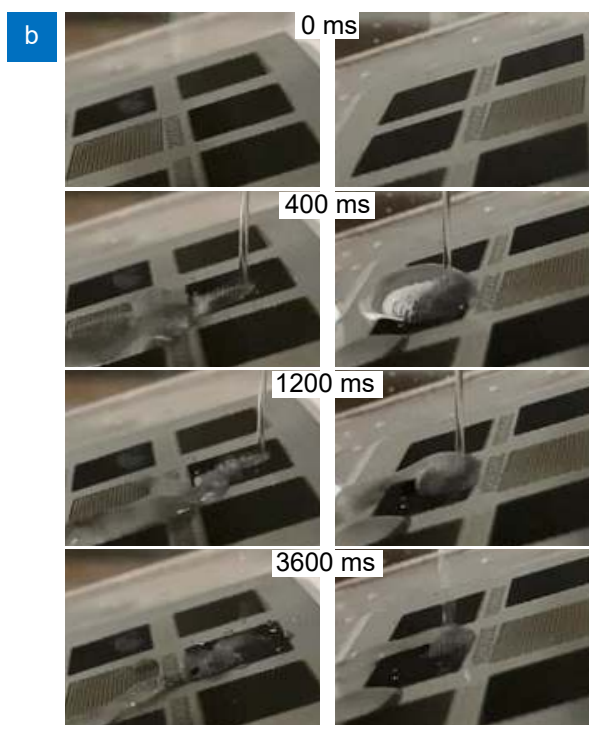

Positive direction
Negative direction

Fig. 3 | Directional water transport property and water repellency of the superhydrophobic surface. (a) Time sequences of snapshots of a water droplet rolling on the as-prepared surface along the positive and negative directions. See Movies S2 and S3. (b) Time sequences of snapshots of water stream flowing through the as-prepared surface along the positive and negative directions. See Movies S4 and S5. The as-prepared surfaces in (a) and (b) are tilted $30^{\circ}$. 

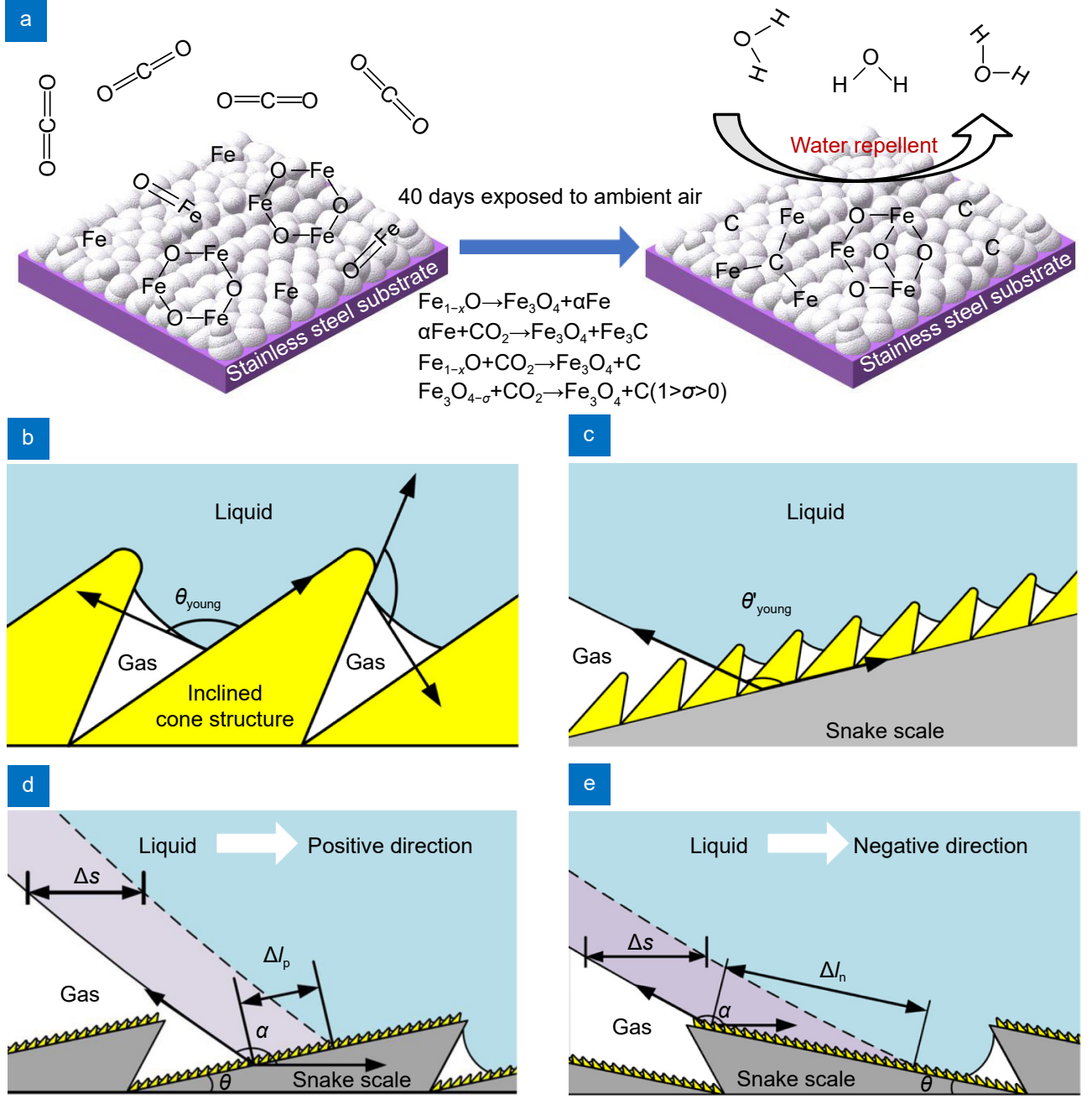

Fig. 4 | Mechanism of directional water transport. (a) Schematic illustration of the molecule/atom interaction mechanism. (b) The intrinsic contact angle on snake scale. (c) The apparent contact angle on snake scale. (d) Schematic illustration of liquid flow in the positive direction of snake scale-like structures. (e) Schematic illustration of liquid flow in the negative direction of snake scale-like structures.

as-prepared surface is tilted at the same angle. Under the action of gravity force, the water flow is accelerated at the moment leaving the syringe and falls to the as-prepared surface. In the experiment, the surface is tilted $30^{\circ}$. Figure 3(b) shows the motion of water flow on the anisotropic surface at different time. Water flows smoothly along the positive direction of the superhydrophobic surface. Under the same conditions, only when the water accumulates and the gravitational potential energy exceeds the adhesion, the water begins to flow along the negative direction of the superhydrophobic surface. Moreover, when the gravitational potential energy drops smaller than the adhesion force by lowering the height of water flow, instead of flowing down along the negative direction of the as-prepared surface, most of the water flow is guided horizontally to both sides by the surface until it exceeds the superhydrophobic surface boundary. More details of the droplets and water flow moving along the two directions are shown in Movies S2 to S5. In these Movies, it is easy to observe the difference between the droplets/flow sliding along the positive and negative directions of the as-prepared surface. The experimental result shows that the adhesion of the liquid sliding against the positive direction is larger than that along the positive direction. Therefore, it can be concluded that different droplet transportation paths can be achieved by using the snake scale-like three-level hierarchical micro/nanostructures to control the water-sliding directivity. Furthermore, it needs to be stressed that droplet transportation just represents one function, the surface with such an ability can be easily extended to other microstructure-dependent functional applications, such as pipeline transportation, vehicle acceleration, and other fluidic devices. 
From the above experimental results, it can be seen that the hierarchies of surface micro/nanostructures (three-level hierarchical snake scale-like micro/nanostructures) and suitable arrangement of the micro/nanostructures (tilted cross stacked arrangement) are important for the smart wetting performance, which can be explained as follows. As a three-level hierarchical structure, the snake scale-like structure consists of large snake scale structures in sub-millimeter scale and inclined coneshaped structures in microscale, decorated with nanoparticles (Fig. 4). First of all, at the nanoscale (Fig. 4(a)), pulsed laser ablation produces active magnetite $\left(\mathrm{Fe}_{3} \mathrm{O}_{4-\sigma}\right.$ with $0<\sigma<1)$ on the surface of stainless steel, which has been identified as an environment capable of dissociating and absorbing carbon dioxide ${ }^{40-43}$. Over time, the carbon dioxide decomposition reaction is sustained and slow under ambient air with nonpolar carbon deposited on the nanoparticles (over 1 month). The efficient amount of low surface energy of carbon and its compound which were deposited on the surface could reduce the surface energy of the as-prepared surface. Thus, the intrinsic contact angle $\theta_{\text {young }}$ of the snake scale-like surface can be increased. Secondly, to combine nanoparticles with microscale inclined cone-shaped structures, there is an opportunity to achieve the high apparent contact angle $\theta_{\text {young }}^{\prime}$ and the Cassie-Baxter state simultaneously (Figs. 4(b) and 4(c), the micro/nanostructures can form air pockets to trap air and lead to the surface superhydrophobicity ${ }^{44}$.

$$
\cos \theta_{\text {young }}^{\prime}=f_{\mathrm{s}} \cos \theta_{\text {young }}-f_{\mathrm{g}},
$$

where $f_{\mathrm{s}}$ and $f_{\mathrm{g}}$ are the fractions of the solid surface and air in contact with water, respectively (i.e., $f_{\mathrm{s}}+f_{\mathrm{g}}=1$ ). Based on the above explanation, it would be easy to understand the transform of surface wetting to superhydrophobic. Meanwhile, after the introduction of the submillimeter scale snake scale-like structures with an inclination angle (Figs. 4(d) and 4(e)), the as-prepared surface acquires anisotropic friction characteristics. When a water droplet is wetting and rolling on the surface, the solid/liquid contact area increases in the forward and decreases in the backward directions. The resistance of the droplet mainly comes from the adhesion force when the liquid detaches from the solid in the backward direction. The friction force of the droplet is related to the detaching area of the solid/liquid interface when the liquid moves a unit distance, and the friction force is the product of interfacial energy and the detaching area di- vided by the unit moving distance $F=\left(\gamma_{\mathrm{gl}}+\gamma_{\mathrm{gs}}-\gamma_{\mathrm{sl}}\right)$ $\times k \times \Delta l / \Delta s^{45}$. Here $\gamma$ is the interfacial energy, $k \times \Delta l$ is the detaching area, $k$ is the width of the liquid flow, $\Delta l$ is the detaching distance of the solid-liquid interface, and $\Delta s$ is the unit moving distance of the liquid droplet. When the liquid droplet rolls in the positive or negative direction of the snake scale-like structures, the ratio of $\Delta l$ to $\Delta s$ is different due to different inclination angles. $\Delta l_{\mathrm{p}} / \Delta s=\sin \alpha / \sin (\alpha-\theta), \quad \Delta l_{\mathrm{n}} / \Delta s=\sin \alpha / \sin (\alpha+\theta)$, where $\theta$ is the inclination angle of the snake scale-like structure, and $\alpha$ is the contact angle. As a result, the detaching distance of the solid/liquid interface $\Delta l_{\mathrm{n}}$ (along the negative direction) is larger than the detaching distance of the solid/liquid interface $\Delta l_{\mathrm{p}}$ (along the positive direction). Therefore, the liquid droplet rolls in the negative direction, the friction force is larger than that in the positive direction. Meanwhile, when the water flow is against the positive direction of the as-prepared surface and the impulse of the flow is large enough, it is easy for the water flow to break the air pockets at the interface and the trapped air in the oblique grooves is replaced by water. This causes the transition of the superhydrophobic surface from Cassie-Baxter to Wenzel state, and the motion state of water flow changes from rolling state to pinning state and produces high adhesion. Therefore, the snake scale-like surface with particular three-level hierarchical micro/nanostructures can realize superhydrophobic and directional tunable water sliding.

Moreover, based on theoretical analyses, the rolling movement of water droplets along the positive and negative directions of the snake scale surface under the action of gravity force is simulated. Simulation of the flowfield is performed using a VOF method with ANSYS Fluent, which is a numerical technique used to track the fluid-fluid interface in computational fluid dynamics ${ }^{46}$. The volume of the water droplet is $20 \mu \mathrm{L}$ and the tilt angle of the snake scale-like surface is $30^{\circ}$. Figures 5 (a) and $5(\mathrm{~b})$ show the simulation models as the droplets rolling in the positive direction and negative direction, respectively. The green, white, and blue areas represent water droplets, air, and substrate, respectively. To simulate the fluid interface between the air and the water. The edge of the water droplet is used as the initial interface. The edge of the rough structure of the substrate is used as the wetting wall. The CA of the wetting wall is $159^{\circ}$. The transient results of the water droplet moving in $16 \mathrm{~ms}$ are calculated. As shown in Figs. 5(c), 5(e) and 5(g), the change 
a

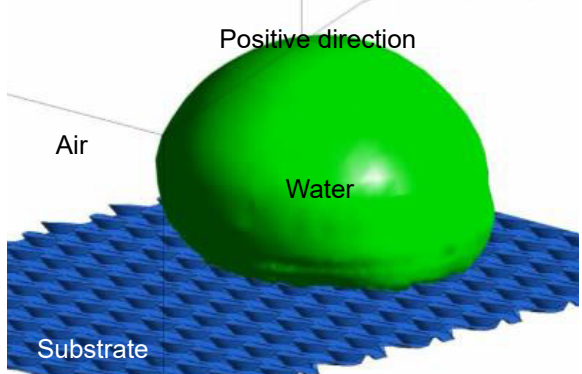

Velocity vector
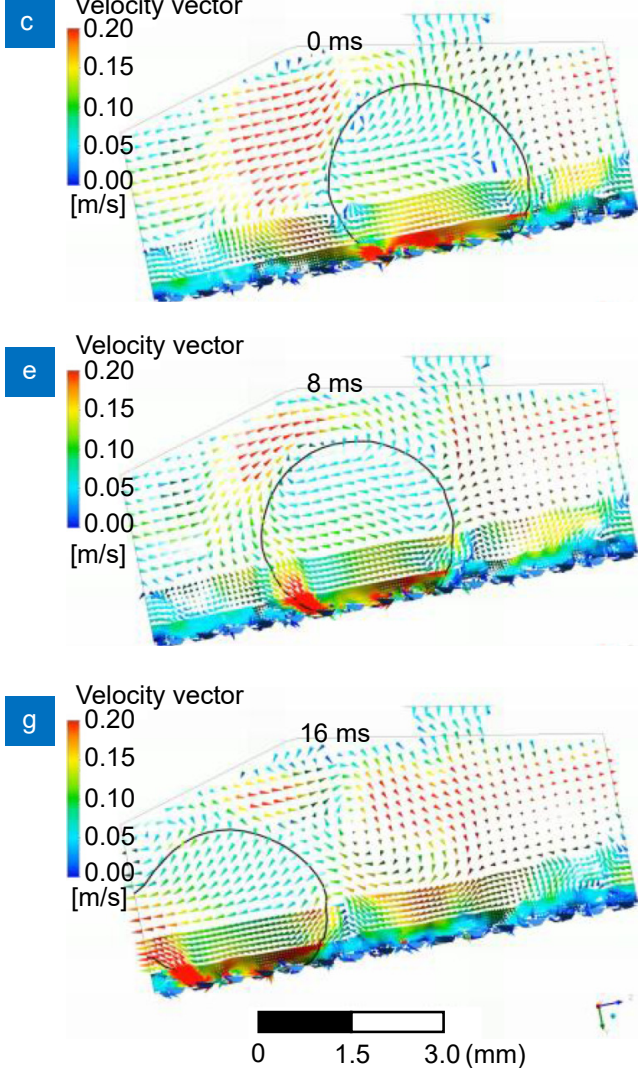

b

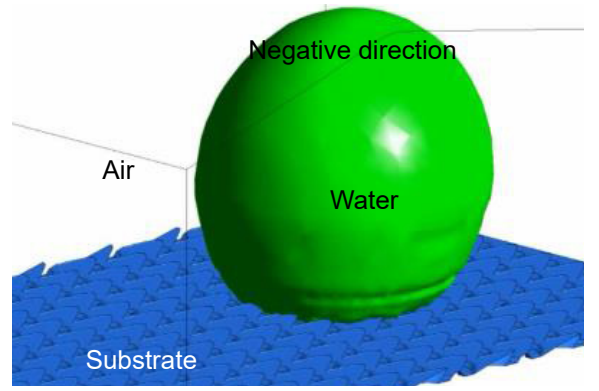

d

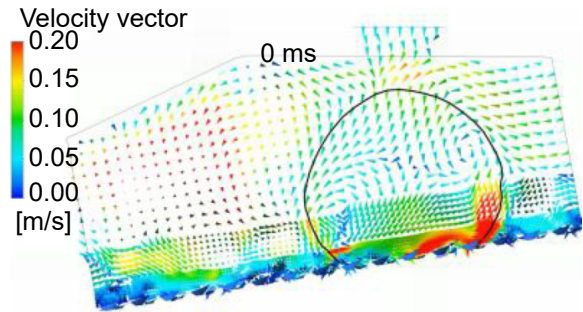

Velocity vector

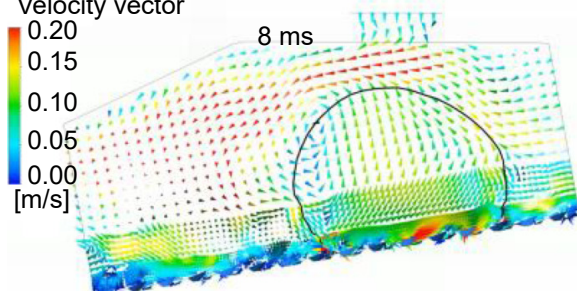

h

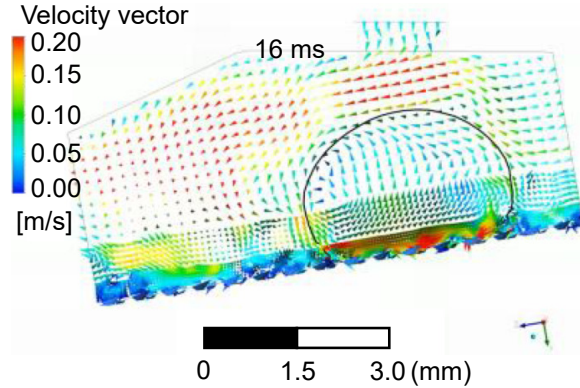

Fig. 5 | Simulated regimes of the droplet sliding on the snake scale-like surface along the positive and negative directions. (a, b) Local magnification of the simulation structures. (c, e, g) Time sequences of simulations of a water droplet rolling on the as-prepared surface along the positive direction. See Movie S6, Supplementary information. (d, f, h) Time sequences of simulations of water droplet rolling on the as-prepared surface along the negative direction. See Movie S7, Supplementary information.

of color scale on the left represents the change of the velocity vector, when the water droplet drops on the snake scale-like surface which is tilted $30^{\circ}$ along the positive direction, the droplet moves about $5 \mathrm{~mm}$ to the left at 16 ms. Correspondingly, when the snake scale-like surface is tilted $30^{\circ}$ along the negative direction, the droplets adhere to the surface and do not move, as shown in Figs. 5(d), 5(f) and 5(h). More results of simulation at different directions are presented in Movies S6 and S7 (see Supplementary information). Under the equal inclination angle of the surface, the simulation results show that the rolling distance of droplet along the positive direction is greater than that along the negative direction, in- dicating that the friction force at the solid-liquid interface along the positive direction is less than that in the negative direction. It is theoretically proved that the snake scale-like surface can realize directional tunable water sliding.

Based on both the excellent superhydrophobicity and high directional performance of the snake scale-like structure surface, it is highly expected that coupling this structure with ship surfaces to form a "ship skin" could further realize directional reducing/increasing friction. The dynamic response between the solid and liquid interfaces under continuous water flow is studied. In particular, stainless steel plates textured with snake 
scale-like structures are designed as skins covered both sides of a ship model to study the directional movement, the water circulation system provides a continuous water flow, and friction force occurs when the surface of the ship moves under the continuous water flow (Fig. 6(a)). As shown in Fig. 6(b) and Movie S8 (see Supplementary information), the ship moves rapidly under the continuous water flow along the positive direction of the as-prepared surface, which is mostly attributed to the directional superhydrophobicity caused by the inclined hierarchical snake scale-like structures. As the water flow is along the positive direction, it is difficult for the water to enter the grooves because of the existence of the scales and the inclined grooves. Additionally, the moving states of the ship can be manipulated by rotating the ship 180 degrees (Fig. 6(c)). The results show that when other conditions are the same as that before the ship rotates, the ship hardly moves with the flow of water, it is

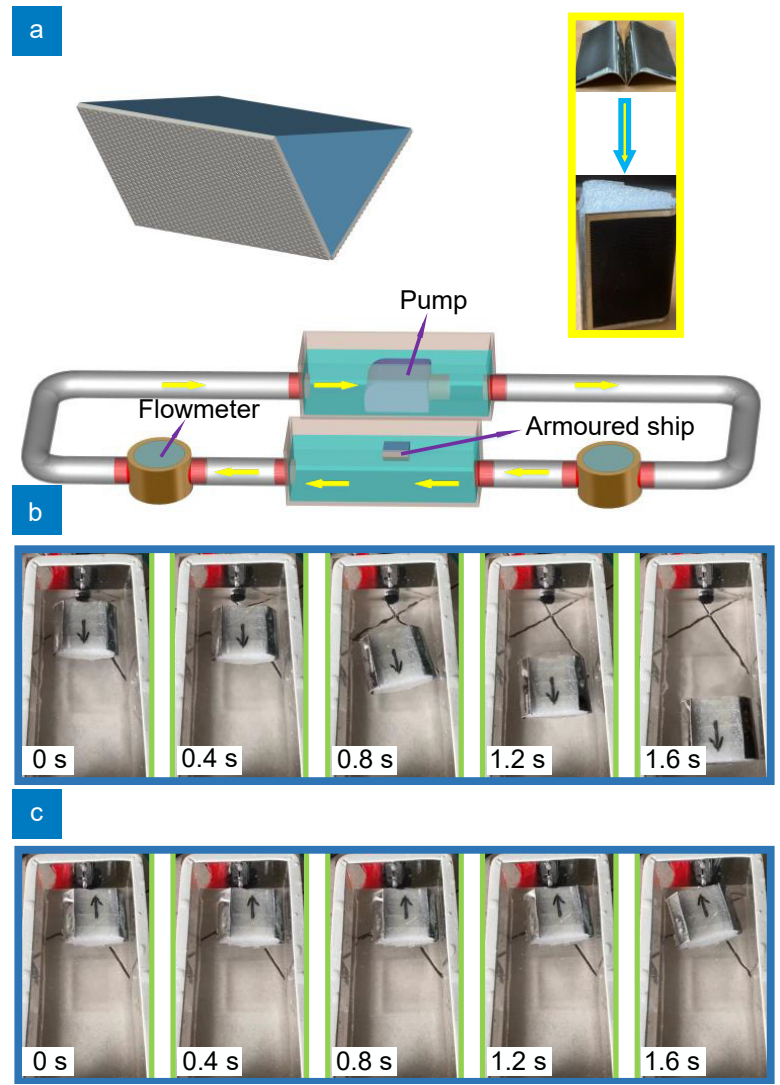

Fig. 6 | Application of the surface as a "ship skin" for controllable navigational direction. (a) The simulated model and the real object of the ship, and the schematic illustration of the water circulation system. (b, c) Time sequences of snapshots of an armoured ship moving under the continuous water flow along the positive and negative directions of the as-prepared surface. See Movie S8, Supplementary information. because the friction force of the as-prepared surface in the negative direction is larger than that in the positive direction, and the air pockets at the interface are broken by the water flow, which causes the increase of surface adhesion force, as explained before. The snapshots of the movement of the ship confirm that the directional superhydrophobic behaviors are achieved by the snake scalelike structures fabricated by the laser processing.

\section{Conclusions}

In conclusion, we present a novel bionic snake scale-like three-level hierarchical micro/nanostructured surface to realize superhydrophobicity and directional water sliding. To demonstrate the unique directional superhydrophobicity controlling, a stainless steel surface with snake scale-like three-level hierarchical micro/nanostructures is fabricated by laser ablation. Experimental results reveal that the CA can be achieved as high as $159^{\circ}$. Moreover, the adhesion force and the SA show strong dependence on the direction of the structures of the surface, giving the possibility of controllable superhydrophobic states along the positive and negative directions. Based on these unique properties, a "ship skin" for directional movement study is proposed. This work can provide new understandings toward solving the problems of superhydrophobic surfaces with directional wettability, and control the water mobility for a wide range of potential applications, such as water harvesting, droplet manipulation, pipeline transportation, and vehicle acceleration.

\section{References}

1. Feng L, Li S, Li Y, Li H, Zhang L et al. Super-hydrophobic surfaces: from natural to artificial. Adv Mater 14, $1857-1860$ (2002).

2. Feng $X J$, Jiang L. Design and creation of superwetting/antiwetting surfaces. Adv Mater 18, 3063-3078 (2006).

3. Zorba V, Stratakis E, Barberoglou M, Spanakis E, Tzanetakis $P$ et al. Biomimetic artificial surfaces quantitatively reproduce the water repellency of a lotus leaf. Adv Mater 20, 4049-4054 (2008).

4. Sanchez C, Arribart H, Guille MMG. Biomimetism and bioinspiration as tools for the design of innovative materials and systems. Nat Mater 4, 277-288 (2005).

5. Gao XF, Jiang L. Water-repellent legs of water striders. Nature 432, 36 (2004).

6. Zheng YM, Bai H, Huang ZB, Tian XL, Nie FQ et al. Directional water collection on wetted spider silk. Nature 463, 640-643 (2010).

7. Zhang DS, Ranjan B, Tanaka T, Sugioka K. Carbonized 
hybrid micro/nanostructured metasurfaces produced by femtosecond laser ablation in organic solvents for biomimetic antireflective surfaces. ACS Appl Nano Mater 3, 1855-1871 (2020).

8. Lou S, Guo XM, Fan TX, Zhang D. Butterflies: inspiration for solar cells and sunlight water-splitting catalysts. Energy Environ Sci 5, 9195-9216 (2012).

9. Parnell AJ, Bradford JE, Curran EV, Washington AL, Adams G et al. Wing scale ultrastructure underlying convergent and divergent iridescent colours in mimetic Heliconius butterflies. J $R$ Soc Interface 15, 20170948 (2018).

10. Spinner M, Gorb SN, Balmert A, Bleckmann H, Westhoff G. Non-contaminating camouflage: multifunctional skin microornamentation in the west african gaboon viper (Bitis rhinoceros). PLos One 9, e91087 (2014).

11. Zheng L, Zhong YH, Gao YH, Li JY, Zhang ZH et al. Coupling effect of morphology and mechanical properties contributes to the tribological behaviors of snake scales. J Bionic Eng 15, 481-493 (2018).

12. Ball P. Engineering shark skin and other solutions. Nature 400, 507-509 (1999).

13. Lu Y, Sathasivam S, Song JL, Crick CR, Carmalt CJ et al. Robust self-cleaning surfaces that function when exposed to either air or oil. Science 347, 1132-1135 (2015).

14. Zang DM, Zhu RW, Zhang W, Yu XQ, Lin L et al. Corrosionresistant superhydrophobic coatings on mg alloy surfaces inspired by lotus seedpod. Adv Funct Mater 27, 1605446 (2017).

15. Chen FZ, Lu Y, Liu X, Song JL, He GJ et al. Table salt as a template to prepare reusable porous PVDF-MWCNT foam for separation of immiscible oils/organic solvents and corrosive aqueous solutions. Adv Funct Mater 27, 1702926 (2017).

16. Golovin K, Dhyani A, Thouless MD, Tuteja A. Low-interfacial toughness materials for effective large-scale deicing. Science 364, 371-375 (2019).

17. Lai YK, Huang JY, Cui ZQ, Ge MZ, Zhang KQ et al. Recent advances in $\mathrm{TiO}_{2}$ - based nanostructured surfaces with controllable wettability and adhesion. Small 12, 2203-2224 (2016).

18. Liu YB, Gu HM, Jia Y, Liu J, Zhang H et al. Design and preparation of biomimetic polydimethylsiloxane (PDMS) films with superhydrophobic, self-healing and drag reduction properties via replication of shark skin and SI-ATRP. Chem Eng J 356, 318-328 (2019).

19. Zhuang AY, Liao RJ, Lu Y, Dixon SC, Jiamprasertboon A et al. Transforming a simple commercial glue into highly robust superhydrophobic surfaces via aerosol-assisted chemical vapor deposition. ACS Appl Mater Interfaces 9, 42327-42335 (2017).

20. Zhu YZ, Wang JL, Zhang F, Gao SJ, Wang AQ et al. Zwitterionic nanohydrogel grafted PVDF membranes with comprehensive antifouling property and superior cycle stability for oil - in - water emulsion separation. Adv Funct Mater 28, 1804121 (2018).

21. Ellinas K, Chatzipetrou M, Zergioti I, Tserepi A, Gogolides E. Superamphiphobic polymeric surfaces sustaining ultrahigh impact pressures of aqueous high - and low - surface - tension mixtures, tested with laser - induced forward transfer of drops. Adv Mater 27, 2231-2235 (2015).

22. Tang M, Hong MH, Choo YS, Tang Z, Chua DHC. Super-hy- drophobic transparent surface by femtosecond laser micro-patterned catalyst thin film for carbon nanotube cluster growth. Appl Phys A 101, 503-508 (2010).

23. Tang M, Shim V, Pan ZY, Choo YS, Hong MH. Laser ablation of metal substrates for super-hydrophobic effect. J Laser Micro/Nanoeng 6, 6-9 (2011).

24. Zhou R, Lin SD, Shen F, Khew SY, Hong MH. A universal copper mesh with on-demand wettability fabricated by pulsed laser ablation for oil/water separation. Surf Coat Technol 348, 73-80 (2018).

25. Yan HP, Rashid MRBA, Khew SY, Li FP, Hong MH. Wettability transition of laser textured brass surfaces inside different mediums. App/ Surf Sci 427, 369-375 (2018).

26. Wang DH, Sun QQ, Hokkanen MJ, Zhang CL, Lin FY et al. Design of robust superhydrophobic surfaces. Nature $\mathbf{5 8 2}$, 55-59 (2020).

27. Cao WT, Feng W, Jiang YY, Ma C, Zhou ZF et al. Two-dimensional MXene-reinforced robust surface superhydrophobicity with self-cleaning and photothermal-actuating binary effects. Mater Horiz 6, 1057-1065 (2019).

28. Song JL, Guan FS, Pan WH, Liu ZA, Sun J et al. Dropletbased self-propelled miniboat. Adv Funct Mater 30, 1910778 (2020).

29. Wang JN, Liu YQ, Zhang YL, Feng J, Wang H et al. Wearable superhydrophobic elastomer skin with switchable wettability. Adv Funct Mater 28, 1800625 (2018).

30. Paradisanos I, Fotakis C, Anastasiadis SH, Stratakis E. Gradient induced liquid motion on laser structured black Si surfaces. Appl Phys Lett 107, 111603 (2015).

31. Kirner SV, Hermens U, Mimidis A, Skoulas E, Florian C et al. Mimicking bug-like surface structures and their fluid transport produced by ultrashort laser pulse irradiation of steel. Appl Phys A 123, 754 (2017).

32. Wu D, Zhang Z, Zhang YY, Jiao YL, Jiang SJ et al. High-performance unidirectional manipulation of microdroplets by horizontal vibration on femtosecond laser-induced slant microwall arrays. Adv Mater 32, 2005039 (2020).

33. Liu XQ, Bai BF, Chen QD, Sun HB. Etching-assisted femtosecond laser modification of hard materials. Opto-Electron Adv 2, 190021 (2019).

34. Ma WZ, Zhao DS, Liu RM, Wang TS, Yuan Q et al. Observation and optimization of $2 \mu \mathrm{m}$ mode-locked pulses in all-fiber net anomalous dispersion laser cavity. Opto-Electron Adv 3, 200001 (2020).

35. Li Y, Hong MH. Parallel laser micro/Nano-processing for functional device fabrication. Laser Photonics Rev 14, 1900062 (2020).

36. Livakas N, Skoulas E, Stratakis E. Omnidirectional iridescence via cylindrically-polarized femtosecond laser processing. OptoElectron Adv 3, 190035 (2020).

37. Jia YC, Wang SX, Chen F. Femtosecond laser direct writing of flexibly configured waveguide geometries in optical crystals: fabrication and application. Opto-Electron Adv 3, 190042 (2020).

38. Stratakis E, Bonse J, Heitz J, Siegel J, Tsibidis GD et al. Laser engineering of biomimetic surfaces. Mat Sci Eng: R: Rep 141, 
100562 (2020).

39. Wenzel RN. Resistance of solid surfaces to wetting by water. Ind Eng Chem 28, 988-994 (1936).

40. Tamaura $\mathrm{Y}$, Tahata M. Complete reduction of carbon dioxide to carbon using cation-excess magnetite. Nature 346, 255-256 (1990).

41. Zhang CL, Li S, Wang LJ, Wu TH, Peng SY. Studies on the decomposing carbon dioxide into carbon with oxygen-deficient magnetite: II. The effects of properties of magnetite on activity of decomposition $\mathrm{CO}_{2}$ and mechanism of the reaction. Mater Chem Phys 62, 52-61 (2000).

42. Zhang CL, Li S, Wang LJ, Wu TH, Peng SY. Studies on the decomposition of carbon dioxide into carbon with oxygen-deficient magnetite: I. Preparation, characterization of magnetite, and its activity of decomposing carbon dioxide. Mater Chem Phys 62, 44-51 (2000).

43. Lau KKS, Bico J, Teo KBK, Chhowalla M, Amaratunga GAJ et al. Superhydrophobic carbon nanotube forests. Nano Lett 3, 1701-1705 (2003).

44. Cassie ABD, Baxter S. Wettability of porous surfaces. Trans Faraday Soc 40, 546-551 (1944).

45. Rowlinson JS, Widom B. Molecular Theory of Capillarity (Clarendon Press, Oxford, Oxfordshire, 1982).

46. Nguyen VT, Park WG. A free surface flow solver for complex three-dimensional water impact problems based on the VOF method. Int J Numer Methods Fluids 82, 3-34 (2016).

\section{Acknowledgements}

This work was supported by the Advanced Remanufacturing and Techno- logy Centre (ARTC) under its RIE2020 Advanced Manufacturing and Engineering (AME) IAF PP Grant (No. A19C2a0019).

\section{Author contributions}

Y. Z. Zhao, and M.H. Hong conceived the research. M. H. Hong and X. Y. Hou supervised the research. Y. Z. Zhao and Y. L. Su carried out the experiment. Y. Z. Zhao and Y. L. Su built the models. All authors analyzed the data and wrote the paper.

\section{Competing interests}

The authors declare no competing financial interests.

\section{Supplementary information}

Supplementary information for this paper is available at https://doi.org/10.29026/oea.2021.210008

Movies S1 to S8

Movie S1. SAs on the as-prepared surface corresponding to the positive and negative directions, respectively.

Movie S2. The variation of the droplet rolling on the as-prepared surface along the positive direction.

Movie S3. The variation of the droplet rolling on the as-prepared surface along the negative direction.

Movie S4. The variation of water flowing through the as-prepared surface along the positive direction.

Movie S5. The variation of water flowing through the as-prepared surface along the negative direction.

Movie S6. The sliding movement of water droplets on the as-prepared surface along the positive direction in simulation.

Movie S7. The sliding movement of water droplets on the as-prepared surface along the negative direction in simulation.

Movie S8. The moving states variation of an armoured ship moving under the continuous water flow along the positive and negative directions of the as-prepared surface. 